\title{
Hepatic Surgeon in the Multidisciplinary Team Managing the Patients with Liver Metastases from Colorectal Cancer - A Prerequisite Condition to Improve Prognosis
}

\author{
Sorin Alexandrescu $\mathrm{u}^{1,2}$, Irinel Popescu ${ }^{1,3}$ \\ "'Dan Setlacec" Centre of General Surgery and Liver Transplantation, \\ Fundeni Clinical Institute, Bucharest, Romania \\ "University of Medicine and Pharmacy "Carol Davila", Bucharest, Romania \\ ${ }^{3}$ Faculty of Medicine, "Titu Maiorescu" University, Bucharest, Romania
}

\section{ABSTRACT}

In patients with colorectal liver metastases (CLMs), liver resection still represents the only potentially curative therapy. However, during the last decade, it was observed that managing the patients with CLMs in a multidisciplinary teams (MDT) will improve survival rates. Although these MDTs represent a prerequisite condition to improve the prognosis of these patients, the presence of a specialized liver surgeon in such structures allows a better allocation of the liver resection in patients with CLMs. Recent evidences revealed that liver surgeons recommended hepatectomy to a significantly higher proportion of patients with CLMs, compared with medical oncologists or other surgeons. Thus, hepatic surgeons will be able to decide the initial resectability of CLMs and to anticipate the potential for future liver resection after oncologic therapy. Therefore, the liver surgeon will contribute to establishing the goal of the treatment at the baseline. Even in patients with initially unresectable CLMs, the liver surgeon should define the criteria for resectability in order to identify as soon as possible the moment when the metastases become resectable. Moreover, an experienced hepatic surgeon will be able to anticipate the risks of postoperative complications based on the duration of preoperative chemotherapy and the volume of future liver remnant, eventually deciding to stop chemotherapy and recommending the performance of portal vein occlusion or of a "two-stage" hepatectomy. The knowledges about the necessity and the role of a hepatic surgeon in an MDT might improve the resectability rates of CLMs and translate in a better prognosis for these patients.

Key words: colorectal liver metastases, multidisciplinary team, hepatic surgeon, liver resection

\section{INTRODUCTION}

The treatment of patients with liver metastases from colorectal cancer (CRC) is multimodal. The available therapeutic options in patients with colorectal cancer liver metastases (CLMs) are liver resection, ablative therapies, cytotoxic drugs (systemic or hepatic artery infusion chemotherapy), targeted therapies, radioembolization (with Yttrium-90) and chemoembolization (irinotecan eluting
Corresponding author:

Sorin Alexandrescu, MD, PhD

Fundeni Clinical Institute

Soseaua Fundeni, 258, sector 2 ,

Bucharest, Romania, 022328

Tel/Fax: 004-0213180417

E-mail: stalexandrescu@yahoo.com

\author{
Abbreviations: \\ OS - overall survival \\ DFS - disease-free survival \\ ORR - overall response rate \\ mCRC - metastatic colorectal cancer \\ CLMs - colorectal liver metastases
} MDT - multidisciplinary team 
beads). The aim of these therapeutic methods is to prolong the patient's survival and even achieve the cure in some cases.

Despite the progresses lately achieved by medical oncology therapy, the highest survival rates are still accomplished by complete resection of CLMs (1). Most retrospective series and data sets revealed that overall survival (OS) rates achieved by liver resection for CLMs were significantly higher than those achieved by palliative treatment (1-3). Some criticisms to this assumption have been launched, because all those data come from retrospective analysis, which compared the long-term outcomes of patients with resectable metastases to those of patients with unresectable CLMs. A recent post-hoc analysis of FIRE-3 trial (4) revealed that the OS of patients with resectable metastases from CRC which were not resected was significantly lower than those of resected patients (30.8 mo. vs. 51.3 mo. $p$ value $<0.001$ ), irrespective the number of metastatic organs. For patients who presented only one metastatic location, the median survival achieved by resection was 64.1 mo., while in similar (resectable) patients who were not resected the median survival was $32.4 \mathrm{mo}$. ( $p$ value $<0.001$ ). Thus, the post-hoc analysis of this randomized trial was able to demonstrate that among the therapeutic options available today, surgery achieves the highest OS rates in patients with metastatic CRC ( $m C R C)$. By this reason, nowadays, the ultimate goal of the treatment in patients with $\mathrm{mCRC}$ should be complete resection of the metastases, because it is obvious that the most important modality to increase the survival rates of patients with CLMs is to increase the rate of resectability.

\section{INDICATIONS OF LIVER RESECTION FOR CLMS}

During the '90s, it was considered that resection of CLMs is worthwhile in patients presenting less than 4 metastases, whose maximum diameter does not exceed $5 \mathrm{~cm}$, in the absence of extrahepatic disease and upon anticipation the ability to achieve a negative margin larger than $1 \mathrm{~cm}$. Later on, data from large registries showed that in patients beyond these criteria, the 5 -year OS rates achieved by liver resection were higher than $20 \%(1)$, obviously superior to the estimated 5 -year OS rates achieved by palliative therapy. Thus, these traditional contraindications to liver resection became gradually just prognostic factors for poor long-term outcomes and their presence is no longer considered a contraindication to liver resection (5). In present, the resectability is defined as the anticipated ability to remove all the metastatic burden ( $\mathrm{R} 0$ resection) with preservation of at least two adjacent liver segments with intact vasculo-biliary inflow and outflow and a volume larger than $25-30 \%$ of total liver volume (6).

\section{RESECTABILITY RATES}

Based on this concept, most series reported by highvolume centers dealing with liver resection for CLMs reveal that almost $20-25 \%$ of patients with CLMs are resectable at the time of initial diagnosis (baseline) $(6,7)$.

Moreover, during the last two decades, there were introduced several onco-surgical approaches able to render to resectability a proportion of the patients with initially unresectable CLMs. The most frequently used modalities to achieve complete clearance of the liver are: 1 . Hypertrophy of the future liver remnant (FLR) by portal vein occlusion (ligation/embolization), two-stage liver resection or associating liver partition and portal vein ligation for staged hepatectomy (ALPPS); 2. Combined resection and ablation of CLMs; 3. Downsizing of liver metastases after oncologic therapy. By employing these strategies, a proportion of $10-40 \%$ of patients with initially unresectable CLMs could become resectable. Thus, in the end, the resectability rates could become as high as $40 \%$.

Unfortunately, these resectability rates presented by high-volume HPB surgical centers were not observed in population-based studies and even in many randomized controlled trials evaluating the efficacy of different oncologic therapies.

Recent retrospective series and post-hoc analysis of randomized trials (4) revealed an actual resectability rate significantly lower than those that would be anticipated after an independent review. These studies unveiled some important issues responsible for this discrepancy between resectability rates among different series. The main findings will be discussed in the present paper, focusing on the role of the surgeon in expanding the proportion of patients that could undergo complete clearance of the liver (either by liver resection or/and ablation).

\section{IMPORTANCE OF THE TREATMENT SETTING AND MULTIDISCIPLINARY TEAM (MDT)}

A retrospective population-based study (including 114155 patients operated for colorectal cancer in UK) revealed that there was significant variation in the rate of liver resection for CLMs across cancer networks 
(range $1 \bullet 1-4 \bullet 3$ per cent) and hospitals (range $0 \bullet 7-6 \bullet 8$ per cent) (2).

A post-hoc analysis of FIRE-3 study (which recruited patients, who were not scheduled for upfront surgery, to receive FOLFIRI plus either cetuximab or bevacizumab) evaluated the resectability rates at baseline and at the best response following oncologic therapy (4). The resectability was reviewed by 8 surgeons and 3 medical oncologists, based on the medical reports and imaging of the patients. Central evaluation revealed that at the baseline $34.7 \%$ (51/147) patients with CLMs were resectable, while at the best response $70.7 \%$ (104/147) patients would be resectable. The actual resection rate of these patients was $25.1 \%$ (37/147), even lower than the initial resection rate. This finding emphasizes the role of an expert liver surgeon to decide the feasibility of a potentially-curative hepatectomy in patients with CLMs (4). Furthermore, the resectability rates were significantly influenced by the treatment setting. Thus, after central review, the estimated resectability rates of all metastatic sites at the best response were similar among university hospitals $(48.7 \%)$, non-university hospitals (55.6\%) and medical practices oncology (52.1\%). However, the actual resection rates were significantly different between university hospitals and other settings ( $p$ value $=0.02$; university hospitals $25.6 \%$, non-university hospitals $-16.9 \%$ and medical practices $-10.4 \%$ ) (4). This difference is most likely due to the difficult access to an expert surgeon's opinion outside university hospitals.

A retrospective analysis of the medical records of all patients with liver-only CLMs who received palliative oncologic treatment during the year 2009 at a regional oncology centre from UK was performed (8). The patients who were discussed in an MDT were excluded. For the remaining 52 patients, the imaging before starting chemotherapy were reviewed by six liver surgeons. In 13 out of 52 patients (25\%) all the six reviewers considered that the liver metastases were resectable, while in another 2 patients all the reviewers estimated that CLMs were likely to become resectable after first-line chemotherapy. Moreover, at least three liver surgeons estimated that additional 18 patients presented potentially resectable CLMs. Thus, 63\% patients were judged to have potentially resectable liver disease, while in only $10 \%$ of the patients the reviewers agree unanimously with palliative treatment (8). This study assessed decision-making by non-liver surgeons in the management of CLMs and demonstrated that, even in a high-volume UK cancer network, almost two-thirds of patients with CLMs deemed unresectable by non-specialists were considered potentially resectable by a panel of specialist liver surgeons (8). The benefit of liver resection in patients with initially unresectable CLMs who were rendered to resectability following oncologic treatment has been demonstrated in PRIME study (9). An analysis of RAS-wt patients enrolled in PRIME study revealed that 2-year OS of patients who underwent complete resection was $96 \%$ vs $41 \%$ in patients who did not undergo complete resection (9).

These facts emphasize the role of MDT which increases the likelihood of CLMs' resection and consequently prolongs survival of the resected patients. Although consensual decision-making within MDT is recommended by guidelines as a contemporary standard in cancer care (10), this seems far from routine clinical practice outside large referral centres (11).

\section{IMPORTANCE OF MDT WITH SPECIALIZED HEPATIC SURGEON}

In a pilot study, 64 specialists dealing with CRC patients (in two university-affiliated academic institutions and two university-affiliated community hospitals in Canada) were asked to judge the resectability of CLMs in 6 clinical cases derived from patients who were actually successfully resected for CLMs (12). Ten of the respondents were liver surgeons, 20 were medical oncologists and 34 were non-hepatobiliary surgeons. While $57 \%$ of the assessments of non-liver surgeons recommended hepatectomy, $92 \%$ of the assessments of liver surgeons deemed the CLMs as resectable ( $p$ value $<0.001$ ). Similar results were reported by other authors (13) who presented a marked discrepancy between medical oncologists and hepatic surgeons regarding the resectability of CLMs. Thus, the concordance between medical oncologists' perception of resectability and the decision on resectability of CLMs by HPB surgeons was $37.7 \%(13)$. The authors concluded that these findings identify potential opportunities to improve education of medical oncologists and management strategies for integrated multidisciplinary care of patients with CLMs (13).

A virtual board meeting with 30 cases was organized at Gottingen, for 11 representative surgeons and 10 medical oncologists (with extensive experience in treatment of $\mathrm{mCRC}$ ) from the northern Germany (11). All the cases actually underwent liver resection for CLMs, but this information was not provided to the participants. The participants were asked to recommend a treatment concept for each case. Surgeons recommended resection of CLMs significantly more often than did medical oncologists. Thus, $75.5 \%$ of the 
assessments performed by surgeons estimated the potential resectability of CLMs, while $61.3 \%$ of medical oncologists' assessments deemed the metastases as potentially resectable $(P<0.001)(11)$. After this first assessment, educational lectures about the role of chemotherapy and surgery in multimodal treatment of CRLM were given. Reports of other cases than those presented during the first assessment were also offered as part of this educational attempt. After that, the same 30 individual cases (presented in a different order) were given for evaluation and the participants were asked to recommend treatment strategy using the same answer options. The participants changed $56.8 \%$ of their initial answers, with significantly more assessments of medical oncologists shifting toward resectability than in surgeons ( $p$ value $=0.033$ ). Despite this increasing option toward resectability, the difference between medical oncologists' and surgeons' assessments in favor of surgery remains significant ( $69 \%$ vs. $85.5 \%, p$ value $<0.001)(11)$. It is worth to be noted that all the medical oncologists invited to this experts' meeting participated previously in clinical trials on $\mathrm{mCRC}$, thus supposing that they already have had robust knowledge in multidisciplinary treatment of these patients.

The above-mentioned observations revealed that the existence of an MDT with medical oncologists and surgeons with limited experience in liver surgery is not able to offer the best therapeutic approach (especially the chance of a potentially curative hepatectomy) to patients with CLMs. The only way to achieve the highest rates of resectability in patients with CLMs is creation of MDTs with participation of experienced hepatic surgeons (8).

Moreover, the decision of medical oncologists to recall for hepatic surgeons' evaluation before starting chemotherapy was only $5 \%$ in patients considered unlikely resectable/convertible after systemic therapy and $36 \%$ in patients deemed potentially resectable after response to systemic therapy (13). This remark underlines that the highest risk of missing the chance of a potentially curative operation is in borderlineresectable patients who might be resected by complex liver operations (e.g. two-stage hepatectomies with/ without portal vein embolization, ALPPS, combined resection and ablation) or in patients likely to become resectable after first-line oncologic therapy.

The above-mentioned assumption may explain the discrepancy between resection rates observed in different clinical trials which enrolled patients with unresectable metastases from CRC. For example, the CRYSTAL trial, as well as the OPUS trial, were completed prior to the widespread implementation of MDT (14). In both trials, although the overall response rates (ORR) in the Cetuximab arm were around $60 \%$, the resectability rates of RAS-wt patients with liver-limited disease were 13.2\% (in CRYSTAL) (14) and 9.8\% (in OPUS) (15). In both studies, the initial evaluation of the patients was performed by medical oncologists, who were also the only specialists involved in the follow-up of these patients (asking for surgical evaluation in selected patients considered as potentially resectable and not in all the patients). In contrast, in CELIM study, whose primary end-point was ORR, the patients were evaluated at the baseline and after 16 weeks by an MDT including liver surgeon (16). Although the ORR was $68 \%$ (similar to those reported by CRYSTAL and OPUS), the RO resection rate was 38\%, more than double than those reported in CRYSTAL and OPUS studies (16). Another phase-two study that included patients with unresectable metastases from CRC (based on the evaluation performed by medical oncologists and liver surgeons from participating centres) revealed an $89 \%$ ORR after initial treatment with FOLFOXIRI and panitumumab (17). Subsequent evaluation during the treatment identified $43 \%$ potentially resectable patients (who underwent surgery) and RO resection could be achieved in $35 \%$ patients (17). Similar results were reported in MetaPan study, which revealed an ORR of $65 \%$ and a $31 \%$ RO resection rate (18). Moreover, POCHER trial (a phase two prospective trial with rate of CLMs resection as primary end point) revealed an ORR of $79.1 \%$ and an RO resection rate of $60 \%$ in 43 patients with initially unresectable CLMs treated with Cetuximab plus chronomodulated irinotecan, 5-fluorouracil, leucovorin and oxaliplatin (19). Thus, although the response rates were similar among different studies, the resectability rates varied widely, depending mainly on the presence/absence of an experienced hepatic surgeon in the MDT dealing with these patients. These evidences demonstrate that the presence of a liver surgeon in the team that evaluated the patients at the baseline and later on during the study period was correlated with higher resectability rates (than those reported by studies which used similar oncologic therapies but did not employed hepatic surgeons in the team that evaluated the patients).

\section{THE ROLE OF THE LIVER SURGEON IN MDT}

Based on the Pan-Asian adapted ESMO consensus guidelines for the management of patients with metastatic colorectal cancer that was published in 2017 
(20), the first question that MDT should answer in patients with newly-diagnosed metastatic colorectal cancer is the goal of the treatment (curative/potentially curative after oncologic treatment/palliative). Thus, it must be decided ab-initio whether the metastases are initially resectable, or not. Even in patients with initially unresectable metastases, sometimes, the clearance of the liver could be achieved by complex surgical procedures such as two-stage liver resection (with portal vein ligation/embolization), ALPPS or association of liver resection with ablation. Although sometimes none of these procedures could be employed, in some patients with oligometastatic disease it is possible to achieve secondary resection after initial oncologic therapy. Thus, an experienced hepatic surgeon is the most adequate person able to establish the initial resectability of CLMs. Even if the metastases are not resectable at the base-line, the anticipation of a potential curativeintent operation after down-sizing of metastases is a crucial information that the liver surgeon should provide to the medical oncologist, because that will help the oncologist in guiding the first-line therapy. Thus, if the goal of therapy is tumor shrinkage, the oncologist will give an intensified first-line regimen, based on the primary tumor location and molecular profile of the tumor (20).

Moreover, the surgeon should establish at that initial moment the criteria for resectability. It was recently revealed that initial definition of criteria for resectability (before starting oncologic therapy) significantly improved resectability rates in patients with initially unresectable CLMs (21).

Another important issue that a liver surgeon should take into account at the baseline is the possibility of disappearance of some small CLMs during first-line therapy. This aspect is tremendously important, because in more than $80 \%$ of cases, radiologic complete response does not mean pathologic complete response (viable tumor cells are usually present) (22). That means that vanishing metastases, which will not be identifiable on imaging, will be very difficult to resect, especially when they are located deep in the liver parenchyma. Thus, at the time of the operation, the surgeon will not be able to resect those malignant foci, leading to a very high likelihood of disease recurrence soon after the operation. By this reason, if the surgeon anticipates such a scenario, he might ask the interventional radiologist to mark the potentially disappearing CLMs with clips. This way, although some metastases would not be visible during the operation, the liver parenchyma around the clips could be resected (under ultrasound guidance), avoiding leaving in place still-viable tumor cells $(23,24)$.

Furthermore, the hepatic surgeon should reassess the metastases every two months, along with medical oncologist and radiologist, in order to establish as soon as possible the potential resectability. The precocious identification of the moment of resectability is also important in order to decrease the risks of postoperative complications. It was demonstrated the hepatic toxicity of prolonged chemotherapy, with Oxaliplatin inducing sinusoidal obstruction and prolonged administration of Irinotecan leading to steatohepatitis. Both of these conditions correlate with increased morbidity rates after major liver resections (which are frequently needed in patients rendered to resectability by oncologic therapy) (25).

The above-mentioned evidence supports the conclusion that a modality to improve the management and prognosis of patients with CLMs is to create colorectal MDTs, including liver surgeons (8). The role of the liver surgeon in MDT are to establish the initial resectability of CLMs, to anticipate the possibility of secondary resection after oncologic therapy, to define the criteria for resectability and identify (as soon as possible) during the oncologic treatment the moment when the metastases become resectable.

\section{Conflict of interest}

All author declare that they have no conflict of interest.

\section{REFERENCES}

1. LiverMet Survey: LiverMetSurvey Statistics December 2015. https://livermet survey manettis.org: 8443/SASStoredProcess/do.

2. Morris EJA, Forman D, Thomas JD, Quirke P, Taylor EF, Fairley L, et al. Surgical management and outcomes of colorectal cancer liver metastases. Br J Surg. 2010;97(7):1110-8.

3. Adam R, Pascal G, Azoulay D, Tanaka K, Castaing D, Bismuth H. Liver resection for colorectal metastases: the third hepatectomy. Ann Surg. 2003;238(6):871-83; discussion 883-884.

4. Modest DP, Denecke T, Pratschke J, Ricard I, Lang H, Bemelmans $M$, et al. Surgical treatment options following chemotherapy plus cetuximab or bevacizumab in metastatic colorectal cancer-central evaluation of FIRE-3. Eur J Cancer. 2018:88:77-86.

5. Popescu I, Alexandrescu ST. Onco-Surgical Management of Liver Metastases from Colorectal Cancer. In: Abdeldayem HM, editor. Updates in Liver Cancer. InTech; 2017. p.115-141.

6. Adams RB, Aloia TA, Loyer E, Pawlik TM, Taouli B, Vauthey J. Selection for hepatic resection of colorectal liver metastases: expert consensus statement. HPB. 2013;15(2):91-103.

7. Adam R, Vinet $E$. Regional treatment of metastasis: surgery of colorectal liver metastases. Ann Oncol. 2004 Oct 1;15(suppl_4): iv103-6.

8. Jones RP, Vauthey J-N, Adam R, Rees M, Berry D, Jackson R, et al. Effect of specialist decision-making on treatment strategies for colorectal liver metastases. Br J Surg. 2012;99(9):1263-9.

9. Douillard J-Y, Siena S, Peeters M, Koukakis R, Terwey J-H, Tabernero J. Impact of early tumour shrinkage and resection on 
outcomes in patients with wild-type RAS metastatic colorectal cancer. Eur J Cancer Oxf Engl 1990. 2015;51(10):1231-42.

10. Lamb BW, Sevdalis N, Mostafid H, Vincent C, Green JSA. Quality improvement in multidisciplinary cancer teams: an investigation of teamwork and clinical decision-making and cross-validation of assessments. Ann Surg Oncol. 2011;18(13):3535-43.

11. Homayounfar K, Bleckmann A, Helms H-J, Lordick F, Rüschoff J, Conradi L-C, et al. Discrepancies between medical oncologists and surgeons in assessment of resectability and indication for chemotherapy in patients with colorectal liver metastases. Br J Surg. 2014;101(5):550-7.

12. Aubin J-M, Bressan AK, Grondin SC, Dixon E, MacLean AR, Gregg $\mathrm{S}$, et al. Assessing resectability of colorectal liver metastases: How do different subspecialties interpret the same data? Can J Surg. 2018;61(4):251-6.

13. Choti MA, Thomas M, Wong SL, Eaddy M, Pawlik TM, Hirose K, et al. Surgical Resection Preferences and Perceptions among Medical Oncologists Treating Liver Metastases from Colorectal Cancer. Ann Surg Oncol. 2016;23(2):375-81.

14. Köhne C-H, Poston G, Folprecht G, Ciardiello F, Ronga P, Beier F, et al. FOLFIRI plus cetuximab in patients with liver-limited or non-liverlimited RAS wild-type metastatic colorectal cancer: A retrospective subgroup analysis of the CRYSTAL study. Eur J Surg Oncol EJSO. 2016:42(10):1540-7.

15. Bokemeyer C, Bondarenko I, Makhson A, Hartmann JT, Aparicio J, de Braud F, et al. Fluorouracil, Leucovorin, and Oxaliplatin With and Without Cetuximab in the First-Line Treatment of Metastatic Colorectal Cancer. J Clin Oncol. 2009;27(5):663-71.

16. Folprecht G, Gruenberger T, Bechstein WO, Raab H-R, Lordick F, Hartmann JT, et al. Tumour response and secondary resectability of colorectal liver metastases following neoadjuvant chemotherapy with cetuximab: the CELIM randomised phase 2 trial. Lancet Oncol. 2010;11(1):38-47.

17. Fornaro L, Lonardi S, Masi G, Loupakis F, Bergamo F, Salvatore $\mathrm{L}$, et al. FOLFOXIRI in combination with panitumumab as firstline treatment in quadruple wild-type (KRAS, NRAS, HRAS, BRAF) metastatic colorectal cancer patients: a phase II trial by the Gruppo Oncologico Nord Ovest (GONO). Ann Oncol. 2013;
24(8):2062-7.

18. Leone F, Artale S, Marino D, Cagnazzo C, Cascinu S, Pinto C, et al. Panitumumab in combination with infusional oxaliplatin and oral capecitabine for conversion therapy in patients with colon cancer and advanced liver metastases: The MetaPan study. Cancer. 2013; 119(19):3429-35.

19. Garufi C, Torsello A, Tumolo S, Ettorre GM, Zeuli M, Campanella C, et al. Cetuximab plus chronomodulated irinotecan, 5-fluorouracil, leucovorin and oxaliplatin as neoadjuvant chemotherapy in colorectal liver metastases: POCHER trial. Br J Cancer. 2010;103(10): 1542-7.

20. Yoshino T, Arnold D, Taniguchi H, Pentheroudakis G, Yamazaki K, $\mathrm{Xu} \mathrm{R}-\mathrm{H}$, et al. Pan-Asian adapted ESMO consensus guidelines for the management of patients with metastatic colorectal cancer: a JSMO-ESMO initiative endorsed by CSCO, KACO, MOS, SSO and TOS. Ann Oncol. 2018;29(1):44-70.

21. Jones RP, Hamann S, Malik HZ, Fenwick SW, Poston GJ, Folprecht G. Defined criteria for resectability improves rates of secondary resection after systemic therapy for liver limited metastatic colorectal cancer. Eur J Cancer. 2014;50(9):1590-601.

22. Benoist S, Brouquet A, Penna C, Julié C, El Hajjam M, Chagnon $\mathrm{S}$, et al. Complete Response of Colorectal Liver Metastases After Chemotherapy: Does It Mean Cure? J Clin Oncol. 2006;24(24): 3939-45.

23. Kornprat P, Schöllnast H, Cerwenka H, Werkgartner G, Bernhardt G, Mischinger H-J. Management of colorectal liver metastases after complete response to neoadjuvant chemotherapy. A case of computertomography-guided wire marking of the liver tumor. Int $\mathrm{J}$ Colorectal Dis. 2009;24(1):125-6.

24. Alonso Casado O, González Moreno S, Encinas García S, Rojo Sebastián A, Olavarría Delgado A. Marcaje de metástasis hepática antes de quimioteria neoadyuvante para su posterior localización y resección mediante hepatectomía no anatómica. Cir Esp. 2013; 91:687-689. Spanish

25. Karoui M, Penna C, Amin-Hashem M, Mitry E, Benoist S, Franc B, et al. Influence of Preoperative Chemotherapy on the Risk of Major Hepatectomy for Colorectal Liver Metastases: Ann Surg. 2006; 243(1):1-7. 